\title{
Analysis of Bacterial Contamination in Blood Bank: Comparison of Two Different Donor Arm Disinfectant
}

\author{
Dr. Nidhya Ganesan ${ }^{1 *}$, Hari Baskar Shanmugham ${ }^{2}$, Dr. Prasanna N Kumar ${ }^{3}$ \\ ${ }^{1}$ Assistant Professor in Pathology, PSG Institute of Medical Sciences and Research, Peelamedu, Coimbatore Tamil Nadu, India \\ ${ }^{2}$ MBBS Student, PSG Institute of Medical Sciences and Research, Peelamedu, Coimbatore Tamil Nadu, India \\ ${ }^{3}$ Professor and HOD, Pathology, PSG Institute of Medical Sciences and Research, Peelamedu, Coimbatore Tamil Nadu, India
}

DOI: $10.36348 /$ SJPM.2019.v04i09.008

| Received: 20.09.2019 | Accepted: 27.09.2019| Published: 30.09.2019

*Corresponding author: Nidhya Ganesan

\section{Abstract}

Bacterial contamination of transfusion products, especially platelets are the most common cause of transfusion associated morbidity and mortality. Minimizing bacterial contamination in blood products is a priority for safe transfusion practices. A prospective study was done comparing the efficiency of two different disinfectant techniques by studying the flora on donor's arm before and after disinfection. This study was undertaken as there are very few reports on this important transfusion protocol from Southern India. Mean number of colonies in post disinfection swab culture with $2 \%$ povidone iodine was found to be greater than that of $2.5 \%$ Chlorhexidine in $75 \%$ isopropyl alcohol. $2.5 \%$ chlorhexidine disinfection offers more effective reduction in bacterial contamination than $2 \%$ povidone iodine group.

Keywords: Chlorhexidine, Povidone iodine, Bacteremia. Copyright @ 2019: This is an open-access article distributed under the terms of the Creative Commons Attribution license which permits unrestricted
use, distribution, and reproduction in any medium for non-commercial use (NonCommercial, or CC-BY-NC) provided the original author and sources
are credited.

\section{INTRODUCTION}

There are reports to indicate that every year India requires 40 million units of blood but only 4 million units are available. Hence the collection and preservation of this precious resource requires utmost care [1]. In India, approximately $57 \%$ of transfusion transmitted reactions have been found to be associated with bacterial contamination of donated blood [2, 3].The French Haemovigilance surveillance system cited bacterial transfusion transmission as the most identified cause of transfusion associated deaths [4].In the UK, between 1995 to 2003, bacterial transfusion transmissions accounted for $58 \%$ of transfusion reactions [5]. Bacteriological screening tests for platelet units were introduced in the US in 2004 and the study showed that the average prevalence of bacterial contamination in platelets from the whole blood was $33.9 / 100,000$ units, that of platelets from apheresis $51 / 100,000$, while that of red cell concentrates was 2.6/100,000[6].

In India, antisepsis with three-step regimen of spirit-10\% povidone iodine-spirit for donor arm is followed currently. The US-FDA has approved use of alcoholic-chlorhexidine as highly effective for donor arm disinfection. However, it is not being used in India. Also, tincture iodine which was used earlier is now discontinued due to its irritant nature [7].
It is observed that Gram positive skin commensals such as coagulase negative Staphylococci and Bacillus cereus are the organisms most often recovered from donated blood which are thought to originate principally through phlebotomy[8]. The introduction of a needle through the skin to collect blood causes the passage of live microbes or the movement of microbes from small skin flaps produced by the needle itself into the collection bags[9].This is found to be effectively reduced by efficient donor arm disinfectants[10].

Approximately $16 \%$ of the deaths due to transfusion reactions have been associated with bacterial contamination, which are usually multi-Drug resistant [7]. Hence, it becomes mandatory to analyse the gaps in the process and implement corrective action to prevent them. SHOT(serious hazards of transfusion), BaCON (Bacterial contamination associated with transfusion) and French haemovigilance surveillance system, studies stated that resident skin flora accounted for $90 \%$ of platelet concentrates and $70 \%$ of red cell concentrates causing transfusion reactions[11,12].

A Blood \&Transplant study conducted by NHS revealed reduction in contamination of blood products by $57 \%$ using improved disinfection strategies [13] 
Review of the literature revealed only a few reports from India on this topic, and since blood safety is a very vital issue, it was thought worthwhile to undertake this study. The aim of this review is to consider the scientific evidence in supporting the efficacy of improved Donor arm disinfection in preventing bacterial contamination of blood.

\section{AIMS \& OBJECTIVES}

The chief objectives of this study are:

- To assess the rate of bacterial contamination in blood bank setting.

- To compare the results from two different donor arm disinfectant techniques.

\section{METHODOLOGY}

This study was a randomised control study using prospective data. 100 subjects were included in the study after calculating the sample size.

The Voluntary donor arrives at the blood bank was tested for eligibility. After obtaining consent form for study, pre-Disinfection sample was collected in sterile swab. Disinfectant was applied for an area of $10 \mathrm{~cm}$ on cubital fossa in concentric pattern. $2.5 \%$ Chlorhexidine in $75 \%$ isopropyl alcohol was applied as disinfectant (group 1) and while 70\% Alcohol followed by $2 \%$ povidone iodine followed by $70 \%$ Alcohol was used as disinfectant (group 2). Post-Disinfection sample was collected in sterile swab. Both the swabs were cultured in blood agar and colony count was performed.

Sterile swabs for the project were made on the same day, supplied by the Department of Microbiology. Samples were collected from the cubital fossa of the donor using sterile forceps by gloved hands and immediately plated on 5\% Sheep blood agar under biosafety cabinets with positive pressure and laminar air flow.

All the patients who have received transfusion from the donors of the sample size were followed up for transfusion reactions. Any allergic reactions were to be notified and treated accordingly. To ensure quality periodical controls for swabs and Disinfectant were established. Technical officers were trained on proper method of disinfection techniques. The waste samples were discarded as per hospital's standard waste disposal system.

Data was entered in Microsoft Excel spreadsheet and analysis was done using SPSS software by Microsoft V.22.0. Association between two factors was expressed in form of odds ratio. $\mathrm{P}<0.005$ was considered significant.

\section{OBSERVATION \& RESULTS}

A total of 98 male and 2 female donors were selected for the study and disinfectant used was randomly assigned to them. Both the pre- and postdisinfection swabs were cultured on blood agar and their efficacy was determined by the number of colonies formed on the blood agar (Table I).

Table-I: Provides the result of swab culture with two disinfectants.

\begin{tabular}{|l|r|c|c|}
\hline DISINFECTANT USED & \multicolumn{1}{l|}{ n } & Colonies-before disinfection & Colonies-after disinfection \\
\hline Povidone iodine & 50 & $40-210$ & $0-10$ \\
\hline Chlorhexidine & 50 & $35-190$ & $0-4$ \\
\hline
\end{tabular}

\section{STATISTICAL ANALYSIS}

Independent student ' $t$ ' test was done to compare the colony count of post-disinfection swab culture between $70 \%$ Alcohol followed by $2 \%$ povidone iodine followed by $70 \%$ alcohol and $2.5 \%$ Chlorhexidine in $75 \%$ isopropyl alcohol . Analysis of covariant was done using General linear model on SPSS 24.0 to compare the mean difference in postdisinfection swab culture adjusted to the bacterial load on pre-disinfection swab culture.
Table II presents the mean (SD) of the postdisinfection swab cultures between the two disinfectants and a statistically significant difference was observed. The mean (SD) of the post-disinfection swab culture for $2.5 \%$ Chlorhexidine in $75 \%$ isopropyl alcohol was 0.2800(0.70102) and 70\% Alcohol followed by $2 \%$ povidone iodine followed by $70 \%$ alcohol was 0.9000(1.97174).t=2.095; $<<0.05$.

Table-II: Comparison of post disinfection swab culture between the two disinfectants

\begin{tabular}{|l|l|r|c|}
\hline DISINFECTANT USED & MEAN & SD & p \\
\hline Povidone iodine & 0.9000 & 1.97174 & $\mathrm{P}<0.05$ \\
\cline { 1 - 3 } Chlorhexidine & 0.2800 & 0.70102 & \\
\hline
\end{tabular}

Analysis of covariant showed that the mean difference of the post-disinfection swab culture adjusted to the bacterial load on pre-disinfection swab culture was statistically significant $F=4.974 ; \mathrm{p}<0.05$.

\section{DISCUSSION}

The hazards of post transfusion septicaemia became less common when closed plastic bags replaced glass containers for collection of blood. Even though it 
has seen a massive decrease, it has not disappeared entirely and there seems to have been a resurgence of interest in it due to modern day increase in genetically improved resistant organisms. It is not always easy to identify the source of bacterial contamination in blood bags. Post transfusion septicaemia can be endogenous which is due to organisms circulating in the blood and in other hand, exogenous post transfusional septicaemia stems from organisms on donors' skin and equipment contamination. Endogenous post transfusional septicaemia is comparatively less than exogenous post transfusional septicaemia due to vigorous donor selection criteria hence extra attention should be given to exogenous post transfusional septicaemia which can be significantly reduced by optimal disinfection of phlebotomy site, since majority of the organisms contaminating the blood products belong to the group of skin commensals[14].

Our skin contains two types of bacterial flora. One is transient flora which can be easily washed away by water and soap. The other is resident flora that requires extensive disinfection with an efficacious disinfectant which we tried to identify in this study.

In our study encompassing 100 donors' disinfection with $2.5 \%$ chlorhexidine in $75 \%$ isopropyl alcohol was comparatively more efficacious than $70 \%$ Alcohol followed by $2 \%$ povidone iodine followed by $70 \%$ alcohol. Mean values of colonies of $70 \%$ Alcohol followed by $2 \%$ povidone iodine followed by $70 \%$ alcohol is greater than what was observed in $2.5 \%$ Chlorhexidine in $75 \%$ isopropyl alcohol in the swab culture of post-disinfection swabs.

We compared our study with similar studies. Lee $\mathrm{C} \mathrm{K}$ et al. reported a relative risk reduction of $3.4 \%$ of chlorhexidine over povidone Iodine in disinfection of donors' arm [15].Ramirez Arcos et al. also observed that disinfection by $70 \%$ alcohol followed by chlorhexidine is highly effective having $98.74 \%$ of reduction rate in bacterial contamination of blood components than what is seen with isopropyl alcohol and iodine which correlates with the results of our study [16]. Also Benjamin RJ et al. proved that the $1.5 \mathrm{ml}$ chlorhexidine disinfection method offers more effective reduction in bacterial contamination of apheresis platelets than $70 \%$ Alcohol followed by $2 \%$ povidone iodine followed by $70 \%$ alcohol [17]

Chlorhexidine is a broad spectrum agent with cumulative actions, which is recommended in the 'Guidelines on drawing blood' by WHO, Centre For Disease Control (CDC) and Health Care Infection Control Practices Advisory Committee (HIPAC) as an optimal skin disinfectant for variety of uses like phlebotomy, vascular catheterization and pre-op preparation of skin[12].
It is also less irritant and non-staining on skin. We found that both the donors and the staff of the blood bank expressed an increased compliance towards it during the period of this study. On application it disrupts the cell wall and is more effective against gram positive organisms than gram negative organisms. In our study we also detected most of the gram positive organisms are inhibited, but due to the small numbers that were studied, analysis and comparison of data cannot be made at this juncture.

A study done by Lee $\mathrm{C} \mathrm{K}$ et al. detected predominantly Bacillus species and Coagulase-negative staphylococci as the most common pathogens [15]. In this study organisms like Coagulase-negative Staphylococci, Micrococci and Diptheroid were mainly isolated in Pre-disinfection swabs, while only Coagulase-negative staphylococci were identified in post- disinfection swabs. This is explained by the emerging resistance of staphylococci to Chlorhexidine [18]. The use of conventional agar plates and gramstaining to identify the morphology of the organism limits the scope of identifying varied bacterial flora which is more evident on automated culture systems like BACTEC/BacTALERT.

This study prompts us to conclude that there is a decrease in bacterial contamination with $2.5 \%$ chlorhexidine in $75 \%$ isopropyl alcohol compared to $70 \%$ alcohol followed by $2 \%$ povidone iodine followed by $70 \%$ Alcohol.

Since there is no significant difference in cost between these two methods tested in our study, Chlorhexidine should be affordable in blood banks of resource limited nations as well.

\section{CONCLUSION}

- Bacterial contamination of blood components remains a major challenge in the practice of transfusion medicine.

- Optimal skin antisepsis of the phlebotomy site is essential to minimize the risk of contamination of collected blood.

- Every reaction of post transfusional septicaemia must be identified, reported, evaluated and the cause for contamination must be identified in order to prevent future post transfusional septicaemia and maintain safe blood practices.

\section{Compliance with Ethical Standards}

\section{Ethical approval}

All procedures performed in studies involving human participants were in accordance with the ethical standards of the institutional and/or national research committee 


\section{Conflict of Interest}

of interest

The authors declare that they have no conflict

\section{Informed consent}

Informed consent was obtained from all individual participants included in the study

\section{REFERENCES}

1. India facing a blood shortage of 3 million units of blood. (2016). Retrieved 21 Nov 2016 from http://timesofindia.com.

2. Patel, T. G., Shukla, R. V., \& Gupte, S. C. (2013). Impact of donor arm cleaning with different aseptic solutions for prevention of contamination in blood bags. Indian Journal of Hematology and Blood Transfusion, 29(1), 17-20.

3. Hoppe, P. A. (1992). Interim measures for detection of bacterially contaminated red cell components. Transfusion, 32(3), 199-201.

4. Debeir, J., Noel, L., Aullen, J. P., Frette, C., Sari, F., Mai, M. P. V., \& Cosson, A. (1999). The French haemovigilance system. Vox sanguinis, 77(2), 77-81.

5. Stainsby, D., Cohen, H., Jones, H., Knowles, S., Milkins, C., Chapman, C., \& Revill, J. (2003). Serious hazards of transfusion (SHOT). SHOT annual report, 1-88.

6. Blajchman, M. A., Ali, A. M., \& Richardson, H. L. (1994). Bacterial contamination of cellular blood components. Vox sanguinis, 67, 25-33.

7. Shah, S., Mehta, N. A., \& Jadhav, S. G. (2014). Prospective evaluation of $2 \%(\mathrm{w} / \mathrm{v})$ alcoholic chlorhexidine gluconate as an antiseptic agent for blood donor arm preparation. Asian journal of transfusion science, 8(2), 92.

8. Leo, A. M., Salvadego, M. M., Piva, M. G., Ruffato, G., Valverde, S., Trabuio, E., ... \& Gessoni, G. (2007). From the donor's arm to blood product: a study on bacterial contamination of apheresis platelet concentrates. Blood Transfusion, 5(3), 130.

9. Stramer, S. L. (2007). Current risks of transfusiontransmitted agents: a review. Archives of pathology \& laboratory medicine, 131(5), 702-707.
10. McDonald, C., McGuane, S., Thomas, J., Hartley, S., Robbins, S., Roy, A., ... \& Barbara, J. (2010). A novel rapid and effective donor arm disinfection method. Transfusion, 50(1), 53-58.

11. Perez, P., Salmi, L. R., Folléa, G., Schmit, J. L., De Barbeyrac, B., Sudre, P., ... \& Bacthem Group the French Haemovigilance Network. (2001). Determinants of transfusion-associated bacterial contamination: results of the French BACTHEM Case-Control Study. Transfusion, 41(7), 862-872.

12. McDonald, C. P. (2011). Interventions implemented to reduce the risk of transmission of bacteria by transfusion in the English National Blood Service. Transfusion Medicine and Hemotherapy, 38(4), 255-258.

13. Dubey, A., Sonker, A., \& Chaudhary, R. (2017). Snake in the grass: A case report of transfusion reactions due to contaminated donor arm disinfectant. Asian journal of transfusion science, 11(1), 50.

14. Puckett, A., Davison, G., Entwistle, C. C., \& Barbara, J. A. (1992). Post transfusion septicaemia 1980-1989: importance of donor arm cleansing. Journal of clinical pathology, 45(2), 155-157.

15. Lee, C. K., Ho, P. L., Chan, N. K., Mak, A., Hong, J., \& Lin, C. K. (2002). Impact of donor arm skin disinfection on the bacterial contamination rate of platelet concentrates. Vox sanguinis, 83(3), 204208.

16. Ramirez-Arcos, S., \& Goldman, M. (2010). Skin disinfection methods: prospective evaluation and postimplementation results. Transfusion, 50(1), 5964.

17. Benjamin, R. J., Dy, B., Warren, R., Lischka, M., \& Eder, A. F. (2011). Skin disinfection with a single-step $2 \%$ chlorhexidine swab is more effective than a two-step povidone-iodine method in preventing bacterial contamination of apheresis platelets. Transfusion, 51(3), 531-538.

18. Goldman, M., Roy, G., Frechette, N., Decary, F., Massicotte, L., \& Delage, G. (1997). Evaluation of donor skin disinfection methods. Transfusion, 37(3), 309-312. 